\section{www.ComputerScienceWeb.com

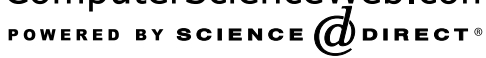

Pattern Recognition Letters 24 (2003) 779-790
Pattern Recognition Letters

www.elsevier.com/locate/patrec

\title{
Segmentation of ultrasound images - multiresolution 2D and 3D algorithm based on global and local statistics
}

\author{
Djamal Boukerroui $^{\mathrm{a}, \mathrm{b}, *}$, Atilla Baskurt ${ }^{\mathrm{c}}$, J. Alison Noble ${ }^{\mathrm{a}}$, Olivier Basset ${ }^{\mathrm{b}}$ \\ a Department of Engineering Science, University of Oxford, Oxford OX1 3PJ, UK \\ ${ }^{\mathrm{b}}$ CREATIS CNRS Research Unit (UMR 5515) and affiliated to INSERM, INSA-502 Villeurbanne Cedex 69621, France \\ ${ }^{\mathrm{c}}$ LIGIM (EA 1899), Claude Bernard University Lyon 1, Villeurbanne Cedex 69622, France
}

\begin{abstract}
In this paper, we propose a robust adaptive region segmentation algorithm for noisy images, within a Bayesian framework. A multiresolution implementation of the algorithm is performed using a wavelets basis and can be used to process both $2 \mathrm{D}$ and $3 \mathrm{D}$ data. In this work we focus on the adaptive character of the algorithm and we discuss how global and local statistics can be utilised in the segmentation process. We propose an improvement on the adaptivity by introducing an enhancement to control the adaptive properties of the segmentation process. This takes the form of a weighting function accounting for both local and global statistics, and is introduced in the minimisation. A new formulation of the segmentation problem allows us to control the effective contribution of each statistical component. The segmentation algorithm is demonstrated on synthetic data, 2D breast ultrasound data and on echocardiographic sequences $(2 \mathrm{D}+\mathrm{T})$. An evaluation of the performance of the proposed algorithm is also presented.
\end{abstract}

(c) 2002 Elsevier Science B.V. All rights reserved.

Keywords: Ultrasound; Bayesian segmentation; Adaptive algorithm; Multiresolution

\section{Introduction}

The first step toward automatic analysis or evaluation of a given image is generally considered to be successful segmentation. In recent years, many authors have applied Bayesian estimation techniques for image segmentation. These statis-

\footnotetext{
${ }^{*}$ Corresponding author.

E-mail addresses: djamal@robots.ox.ac.uk (D. Boukerroui), abaskurt@ligim.univ-lyonl.fr (A. Baskurt), noble@robots. ox.ac.uk (J.A. Noble), olivier.basset@creatis.univ-lyon1.fr (O. Basset).
}

tical approaches have improved the segmentation results of different image modalities (natural scenes (Pappas, 1992; Unser, 1995), texture images (Bouman and Liu, 1991; Kervrann and Heitz, 1994), ultrasonic images (Ashton and Parker, 1995; Boukerroui et al., 1998; Xiao et al., 2000)). The segmentation results usually depend heavily on the estimation of the model region parameters that can be based on local/global statistics and can be adaptive.

Ultrasound images are of relatively poor quality and segmentation is a difficult problem (Ashton and Parker, 1995). The image degradation includes primarily speckle noise, which can be modelled as 
a multiplicative degradation field (Hughes and Duck, 1997), the blurring of spatial information perpendicular to the propagation direction of ultrasonic waves and the non-linear attenuation of ultrasound. When specular structures are imaged, the detected echo amplitude varies according to the orientation of the reflecting structure and therefore the contours can appear discontinuous. Moreover, despite the use of dynamic focusing techniques, the lateral resolution is poor and the structures are often blurred in a direction perpendicular to ultrasonic propagation. Therefore, segmentation algorithms based only on global information (such as thresholding techniques, global clustering approaches and intensity-dependant edge detection) are not suitable and give poor results. This emphasises a need to develop segmentation (or feature detection) techniques that can avoid the intensity inhomogeneity problem or approaches which take into account the non-uniformity of tissue classes. To our knowledge three kinds of solution are proposed in the literature:

- Mulet-Parada and Noble $(1998,2000)$ suggest a phased-based feature detection method. The most important advantage of this technique is its intensity-independence. However, as the noise rejection in this method involves an intensitybased noise threshold the method is not truly intensity invariant and is highly susceptible to noise. Applied to echocardiographic image sequences, the $2 \mathrm{D}+\mathrm{T}$ version of the published technique takes advantage of temporal continuity in order to improve its robustness to noise and detect only relevant and continuous features over time.

- Recently, Xiao et al. (2000) proposed a statistical model that exploits knowledge of tissues properties and intensity inhomogeneities in ultrasound for simultaneous contrast enhancement and segmentation. The underlying model was proposed by Wells et al. (1996) for bias field correction and segmentation of magnetic resonance (MR) images. Results shown for breast and cardiac ultrasound images demonstrate that it can successfully remove intensity inhomogeneities and significant improvement is achieved in tissue contrast and the resulting image segmentation.
Although, this is a promising result, it still requires user interaction to set the image model parameters.

- Recently, following the publication of the adaptive clustering K-means algorithm (Pappas, 1992), several adaptive approaches have been proposed for ultrasound images. Indeed, the adaptive property of the Pappas algorithm is useful because it considers local class mean with a slowly spatial variation, compensating for the non-uniformity of ultrasound echo signals within the same tissue region. Therefore, intensity-based (Ashton and Parker, 1995) and texture-based (Boukerroui et al., 1998, 2001) adaptive Bayesian segmentation approaches taking into account, intrinsically, the non-uniform nature of the tissue classes, have yielded promising results.

In previous work (Boukerroui et al., 1998), we have presented an adaptive segmentation algorithm based on texture characterisation in a Bayesian framework. The underlying model was originally proposed by Ashton and Parker (1995). In the current work we focus on the adaptive character of the algorithm and we discuss how global and local statistics can be utilised during the segmentation process. In other words, the paper proposes an improvement of the adaptivity by introducing a function to control the adaptive properties of the segmentation process. A weighting function, taking into account both local and global statistics is used during the segmentation process. This new formulation of the segmentation problem allows us to control the effective contribution of each statistic.

The paper is organised as follows. After a description of the mathematical framework of our previous work, the proposed improvement on the adaptive character of the algorithm is presented in Section 2. A brief description of the algorithm and implementation details are given in Section 3. Section 4 presents segmentation results on synthetic data, 2D ultrasound breast images and echocardiographic images $(2 \mathrm{D}+\mathrm{T})$. In the case of echocardiographic data a comparison of the computer-generated boundaries of the left ventricle with the hand-outlined contours drawn by a 
medical expert is presented. Results obtained with Xiao et al. (2000) segmentation method are also presented for comparison. The paper's conclusions are summarised in Section 5.

\section{Segmentation method}

In this section we briefly outline the adaptive texture-based clustering algorithm. The reader may refer to (Boukerroui et al., 1998, 2001; Ashton and Parker, 1995) for further details.

We assume that the observed data $Y$ is a random field defined on a 2D (or 3D) isotropic rectangular grid $S$. $Y_{s}$ denotes the value of $Y$ at the site $s \in S$. A segmentation of the image (volume) into regions will be denoted by $X$, where $X_{s}=i$ means that the pixel (voxel) at $S$ belongs to region $i$. The probability $P(X=x)$ is written as $P(x)$. The number of different regions in $X$ is $k$. The conditional density function of $Y$ given $X$ is assumed to exist and to be strictly positive and is denoted by $P(y \mid x)$. The image may be segmented by estimating the pixel classification $X$ given the observed image $Y$ using the maximum a posteriori criteria. We use a Markov random field (MRF) to model the region process $X$, due to its restriction to local interaction. With this assumption, according to Hammersley-Clifford theorem (Besag, 1974), and for a given neighbourhood system, the prior density $P(x)$ can be written as a Gibbs density where

$\ln P(x) \cong \sum_{c \in C} V_{c}(x)=\sum_{\langle s, t\rangle} \beta_{\langle s, t\rangle}\left(1-2 \delta\left(x_{s}, x_{t}\right)\right)$.

Here, $V_{c}(x)$ are the clique potentials and $\delta$ is the Kronecker delta function.

We use an 8-connected (6-connected) spatial neighbourhood for 2D (3D) lattice. $\beta_{\langle s, t\rangle}=\beta$ if the clique $\langle s, t\rangle$ is horizontal or vertical and $\beta_{\langle s, t\rangle}=\beta /$ $\sqrt{2}$ if it's right or left diagonal. $\beta$ is a positive parameter, so that two neighbouring pixels are more likely to belong to the same class than to different classes.

The conditional density distribution $P_{s}\left(y_{s} \mid x_{s}=i\right)$ of the observed grey level intensity at a site $s$ is assumed to be Gaussian, with mean $\mu_{s}^{i}$ and vari- ance $\left(\sigma_{s}^{i}\right)^{2}$. The local class mean $\mu_{s}^{i}$ is a slowly varying function of $s$. These assumptions lead to the following posterior energy function:

$$
U(x \mid y)=\sum_{s}\left\{\ln \left(\sigma_{s}^{x_{s}}\right)+\left(\frac{y_{s}-\mu_{s}^{x_{s}}}{\sqrt{2} \sigma_{s}^{x_{s}}}\right)^{2}\right\}+\sum_{\langle s, t\rangle} V_{\langle s, t\rangle}(x) .
$$

This function has two components. The first term constrains the region intensity to be close to the data and the second is a regularisation term, which imposes a smoothness constraint. To improve the robustness of the algorithm, in our earlier work (Boukerroui et al., 1998) we have modified the energy function (2) by adding other constraints based on parametric measurements that are representative of each region.

Let $\left\{Y_{1}, \ldots, Y_{p}\right\}$ be a set of features calculated on each site of the data. Grey-scale parametric images (volumes) are modelled in the same way as grey scale intensity images in (Pappas, 1992). Using the same notations, the complete energy function takes the following form:

$$
\begin{aligned}
U\left(x \mid y, y_{1}, \ldots, y_{p}\right)=\sum_{s} & \left\{\ln \left(\sigma_{s}^{x_{s}}\right)+\left(\frac{y_{s}-\mu_{s}^{x_{s}}}{\sqrt{2} \sigma_{s}^{x^{s}}}\right)^{2}\right\} \\
& +(p+1) \sum_{\langle s, t\rangle} V_{\langle s, t\rangle}(x) \\
& +\sum_{j=1}^{p} \sum_{s}\left\{\ln \left(\sigma_{j}^{x_{s}}\right)\right. \\
& \left.+\left(\frac{\left(y_{j}\right)_{s}-\left(m_{j}\right)_{s}^{x_{s}}}{\sqrt{2} \sigma_{j}^{x_{s}}}\right)^{2}\right\} .
\end{aligned}
$$

Computation of the exact minima of energy functions ((2) and (3)) is time consuming. As an alternative to simulated annealing (Kirkpatrick et al., 1983), the Iterated Conditional Mode algorithm (Besag, 1986) has been used which does not guarantee a global minimum of the energy function, but is a fast deterministic algorithm.

\subsection{Limitations and discussion}

A major difficulty with version of the algorithm described in (Pappas, 1992; Ashton and Parker, 
1995; Boukerroui et al., 1998), is that it takes into account the global statistics of the regions only in the first few iterations. Indeed, when the algorithm starts from a bad initialisation or when the statistics of regions are not Gaussian the adaptive properties decrease the robustness of the algorithm and prevent it from converging to the optimal solution.

Unser (1995) proposed a different and interesting adaptive extension of the $\mathrm{K}$-means clustering algorithm. The essential difference between this algorithm and Pappas (1992) approach is that Unser utilises a membrane model to specify the smoothness of the means over regions rather than a sliding windows approach. Additionally this technique does not include the MRF regularisation term. The use of a membrane spline regulariser to control the spatial smoothness of the means is an interesting idea as sharp changes of the mean are not expected within the same region. However, the smoothness is controlled by one global parameter for all the regions of the data. Moreover, such a regularisation model (based on a priori information) does not take into account the observed data. Indeed, in the special case of ultrasonic images, the spatial variation of the local means depends on their absolute value because of the multiplicative nature of the degradation.

In the next section, we present a new formulation to control the adaptive properties of the segmentation process which takes into account the observed data. In order to illustrate clearly the enhancement made, the new formulation of the segmentation problem is presented only for grey level data.

\subsection{The proposed energy function}

We propose, in this paper, to introduce a weighting function into the energy function to take into account the global statistics of the image. The energy function becomes:

$$
\begin{aligned}
U(x \mid y)= & \sum_{s} \frac{\varphi\left(W_{s}^{x_{s}}\right)}{N_{s}^{\varphi}}\left\{\ln \left(\sigma_{s}^{x_{s}}\right)+\left(\frac{y_{s}-\mu_{s}^{x_{s}}}{\sqrt{2} \sigma_{s}^{x_{s}}}\right)^{2}\right\} \\
& +\sum_{\langle s, t\rangle} V_{\langle s, t\rangle}(x),
\end{aligned}
$$

where $W_{s}^{x_{s}}$ is a weighting coefficient which measures the similarity between the local statistics of the region $x_{s}$ and the global statistics. $\varphi$ is a transformation function and $N_{s}^{\varphi}$ is a normalisation constant of the function $\varphi$ at the site $s$ defined by the following condition:

$\sum_{x_{s}} \varphi\left(W_{s}^{x_{s}}\right)=\tilde{k}$.

Here, $\tilde{k}$ is the number of regions in the analysing window centred at the site $s$.

In the case of a segmentation problem, the similarity measure that is largely used to compare two distributions is the Kolmogorov-Smirnov distance (Geman, 1990; Muzzolini et al., 1993; Kervrann and Heitz, 1994). So we define $W_{s}^{x_{s}}=$ $D\left(y\left(x_{s}\right), y\left(s, x_{s}\right)\right)$; where $D$ is the KolmogorovSmirnov distance between the cumulative distribution functions of the whole sites of the class $x_{s}$ and the sites of the same class in the analysed window $W$ centred at $s$. The introduction of the transformation function $\varphi$ aims to control the adaptive term $A_{s}$ and the weighting one $W_{s}^{x_{s}}$. We define $\varphi$ as

$\varphi\left(W_{s}^{x_{s}}\right)=A_{s}+\left(W_{s}^{x_{s}}\right)^{b}$

and

$A_{s}=a\left(\max _{\text {all class }}\left[\left(W_{s}^{x_{s}}\right)^{b}\right]-\min _{\text {all class }}\left[\left(W_{s}^{x_{s}}\right)^{b}\right]\right) \quad \forall s \in S$,

where $a$ and $b$ are positive constants.

Thus, at each site $s$, the constant $a$ controls the contribution of the adaptive term $A_{s}$ (local statistics) and the weight $W_{s}^{x_{s}}$ which, takes into account the global statistics. The influence of the control parameters $a$ and $b$ on the $\varphi$ function is illustrated in Fig. 1 (For example for $a \gg 1$, Eq. (4) is equivalent to Eq. (2); see Appendix A). Thus, for large values of the parameter $a$, the weighting function considers mainly the local statistics, and the adaptive character of the algorithm is emphasised. Conversely, for small values of $a(\ll 1)$, the contribution of the local statistics to the labelling decision is relatively low in comparison to the global one, leading to the decreasing of the adaptive property of the algorithm. We use $b=1$ in the following. 


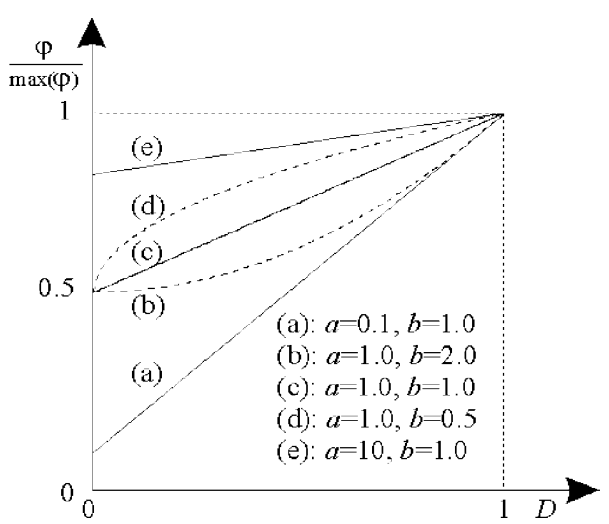

Fig. 1. The influence of the control parameters $a$ and $b . D$ is the Kolmogorov-Smirnov distance.

\section{Algorithm}

In Section 2, the conditional density distribution of the observed grey level intensity at a site $s$ is assumed to be Gaussian. Clearly this assumption is not true in many cases, especially for displayed ultrasound data. As in (Ashton and Parker, 1995) we make use of the Central Limit Theorem which states that the distribution of the mean of a large number of independent random observations tends toward a Gaussian distribution centred on the collective mean. This assumption is reasonably acceptable for low-pass filtered and decimated images that are originally governed by nonGaussian statistics and leads to a multiresolution implementation of our algorithm (Boukerroui et al., 1998).

Starting from the highest resolution image, a multiresolution discrete wavelet transform (DWT) pyramid is built. The coarsest resolution is initially segmented and the result of the segmentation is passed on to the next finer level of resolution and so on, until the finest resolution image is segmented. An initial solution of the minimisation problem, at the coarsest resolution, is obtained with the K-means clustering algorithm. Starting from this segmentation, the algorithm alternates between the estimation of region labels and model parameters and is stopped when no further changes in the labels occur.

A major difficulty with the multiresolution structure is the adhoc choice of the Gibbsian para- meter $\beta$ at each resolution level. The problem of its estimation is studied in (Vemuri et al., 1995) in the special case of the segmentation of brain MR images. The authors propose a prior model for the estimation problem. Some studies (Bouman and Liu, 1991; Pappas, 1992) propose a constant value for parameter $\beta$ whereas Ashton and Parker (1995) suggest adapting its value to each resolution $(\beta$ increases linearly with resolution). In (Heitz et al., 1994), the authors propose to derive the Gibbsian parameter at each scale, directly from the full resolution scale. This method shows clearly that the Gibbsian parameter has a small value at the coarsest resolution and increases, but not linearly. In this paper, the Gibbsian parameter at each scale is derived from the full resolution scale $\left(\beta^{0}\right)$ according to the multiscale approach proposed in (Heitz et al., 1994). The number of regions $k$ and the Gibbsian parameter at the full resolution $\beta^{0}$ are not estimated.

\section{Results}

\subsection{Synthetic data}

The first two experiments were carried out on synthetic data. Synthesised images provide a controlled environment, which allows quantification of the performance of the algorithm because a reference image is available. The aim of these experiments was to evaluate the improvement of the segmentation when the global statistics were taken into account in an adaptive algorithm. Two $128 \times 128$ synthetic images (Fig. 2) were used in the experiments. The image in Fig. 2(a) contains four distinct white Gaussian textures with close means and variances (1: $\mu=180, \sigma=25 ; 2$ : $\mu=120, \sigma=30 ; 3: \mu=150, \sigma=30 ; 4: \mu=150$, $\sigma=20$ ). In Fig. 2(b), the intensities in the image in Fig. 2(a) has undergone a vertical linear attenuation. This is achieved by multiplying the original intensity values by a multiplicative coefficient which is constant horizontally and decreases linearly in the vertical direction.

Fig. 3 shows the segmentation results for the two synthetic images and for three different values of the control parameter $a$. For both images, poor 


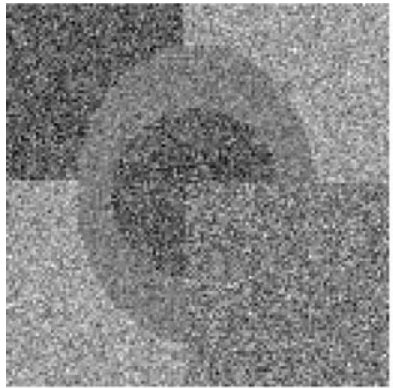

(a)

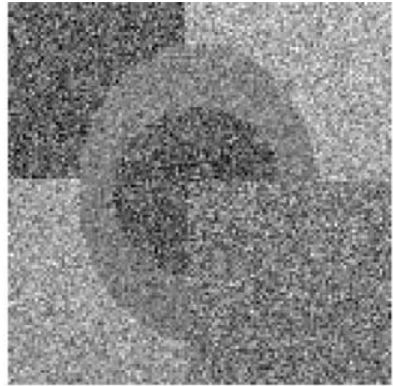

(b)

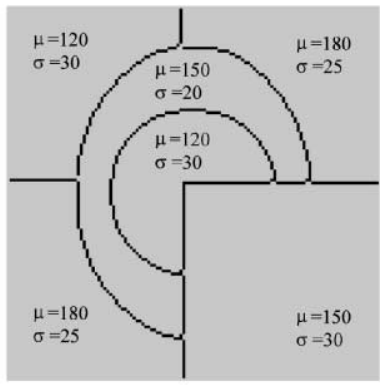

(c)

Fig. 2. Synthetic original images; four white Gaussian textures (a); image (a) with a vertical linear attenuation (1-0.9 from top to bottom) (b); means and standard deviations of the homogeneous regions (c).

segmentation results are obtained if the weighting function considers mainly the local statistics (large value of $a$ ). One can observe that the segmentation results of image (a) for $a=1$ and $a=0.1$ appear in good agreement whereas there is a difference of regions labelling for the segmentation of the image (b). This is because the different regions are homogeneous in image (a). This is not the case in image (b) because of the vertical attenuation. The segmentation result of the image (b) for $a=0.1$ is interesting. Knowing that the degradation field varies vertically, the resulting mean of the upper part (top right) and the lower part (bottom left) of the same region will be slightly different. When the weighting function considers mainly the global statistics $(a=0.1)$ the algorithm classified the upper part and the lower part of this class into two different classes because of the non-homogeneity of the regions whereas it labelled the ring and the right bottom square to the same class. Note that the ring region and the right bottom square have the same mean. An examination of the segmenta- (a)
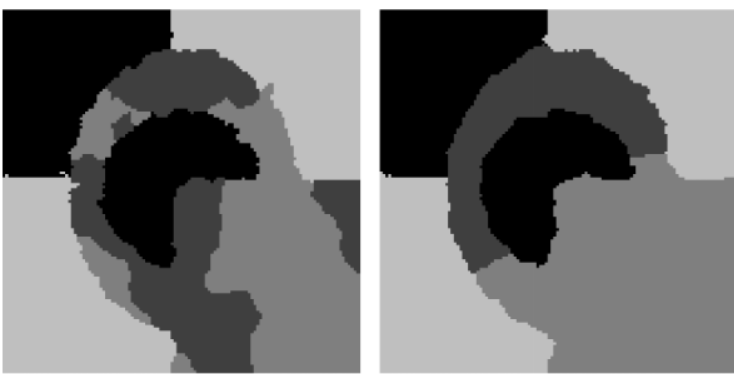

(b)

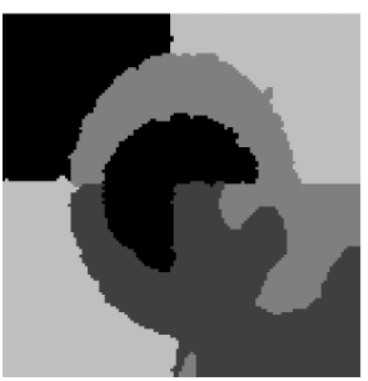

$$
a=10
$$

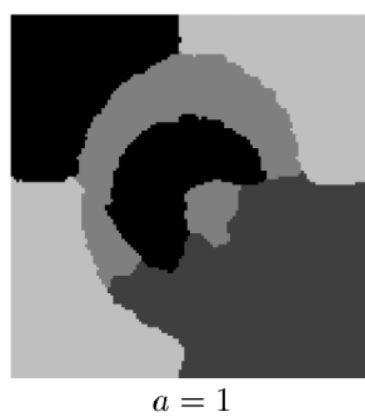

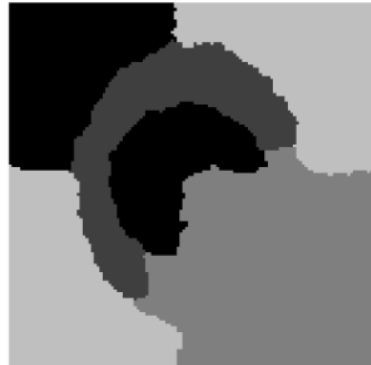

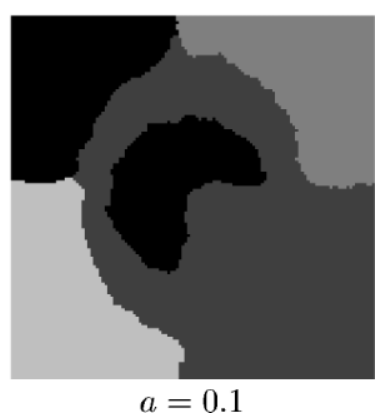

Fig. 3. Segmentation results for $k=4, \beta^{0}=0.3$ and for different values of the control parameter $a$ : image 2(a) (a); image 2(b) (b). 
tion results indicates that $a=1$ gives a good tradeoff between global and local statistics.

The evolution of the segmentation map of the image in (Fig. 2(b)) from coarse to fine resolution with an overestimated number of classes $(k=5)$ is shown in Fig. 4. When the distribution is not homogeneous within the regions (case of Fig. 2(b)), the assumption of a Gaussian distribution for the grey levels is not valid. The initial segmentation provided by the K-means algorithm (Fig. 4(a)) is far from an optimal solution. If we chose to emphasise the adaptive character of the algorithm $(a=+\infty)$, false regions are preserved and the algorithm does not converge toward a satisfying segmentation (Fig. 4(e)). Note how close the result (e) is to the initial solution (a). If instead we chose to take global statistics into account in the weighting function, this leads to less false regions and to a segmentation closer to the optimal solution (Fig. 4(d)). The intermediate results (b) and (c), illustrate the behaviour of the algorithm and the evolution of the segmentation with resolution. The improvement achieved by taking into account the global statistics in the segmentation process is seen by comparing results (d) and (e). However the computation complexity increases with the introduction of the weighting term. For example the processing times on a Pentium 400 $\mathrm{MHz}$ for the result (e), obtained with Ashton and Parker (1995) method is 4 and $16 \mathrm{~s}$ for the results (d) obtained with the new method.

\subsection{Cardiac $2 D+T$ data}

In the third experiment, the application of our new algorithm on a long-axis echocardiographic sequence of 106 images $(2 \mathrm{D}+\mathrm{T})$ is carried out. In the segmentation process the sequence is considered as a $3 \mathrm{D}$ volume to enable temporal continuity to be used in the segmentation process. Results obtained with Xiao et al. (2000) segmentation method are also presented for comparison

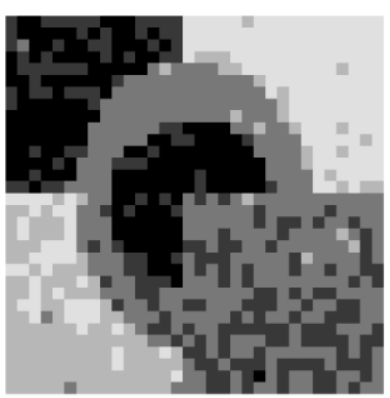

(a)

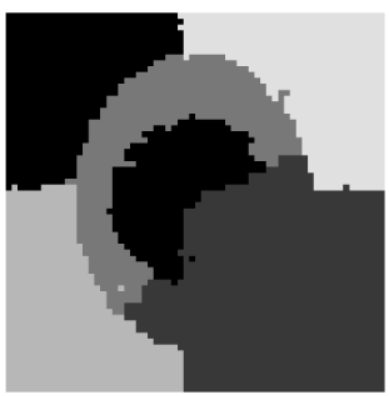

(c)

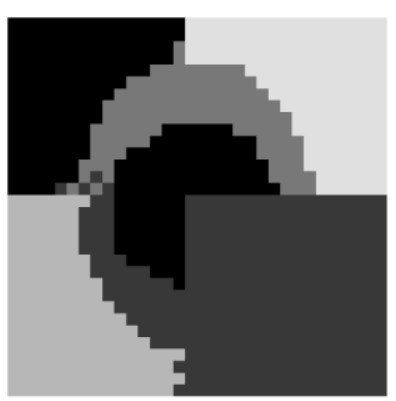

(b)

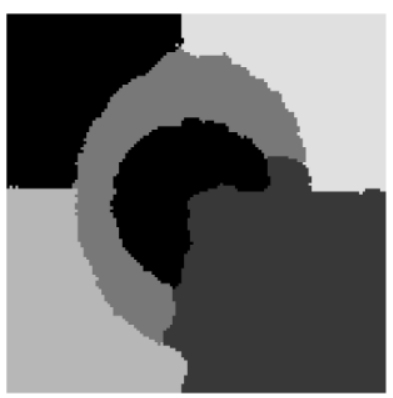

(d)

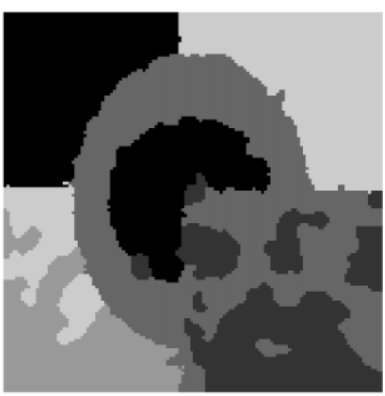

(e)

Fig. 4. Evolution of the segmentation map of the original image Fig. 2(b) from coarse to fine resolution for $k=5, a=b=1$ and $\beta^{0}=0.3$ : initial solution (a); results at the end of resolution 2 (b); at the end of resolution 1 (c); final result (resolution 0) (d). The segmentation result (e) was obtained using the same parameters but for $a=+\infty$ (i.e. using the energy function in Eq. (2)). 
(Fig. 5D). Xiao's approach is semiautomatic, as it requires user interaction to set the image model parameters. The results presented in this paper were obtained after a moderate number of experiments (3-5) to set the image parameters. The Xiao algorithm was run with two classes; the means/ standard deviations were set to $35 / 10$ for the cavity region and 100/40 for the background. The proposed segmentation technique was run with three classes, $\beta^{0}=1$ at the full resolution and for three levels of decomposition of the DWT.
Fig. 5 shows the segmentation results (superimposed on the original data) for four frames (1-4) and for three different values of the control parameter $a(\mathrm{~A}, \mathrm{~B}, \mathrm{C})$. Images $\mathrm{A}$ were obtained for $a=+\infty$ i.e. using the energy function in (2). B and $\mathrm{C}$ were obtained with $a=10$ and $a=1$ respectively. A deviation with respect to the true boundaries is observed in the left part of the images (right ventricle) when the adaptive character is emphasised (Fig. 5A), whereas a good detection was obtained when global statistics are included in the

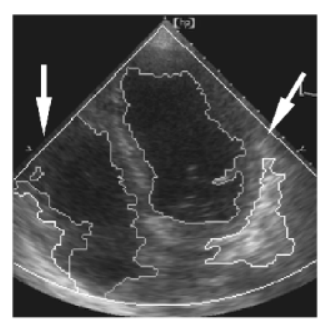

(A1)

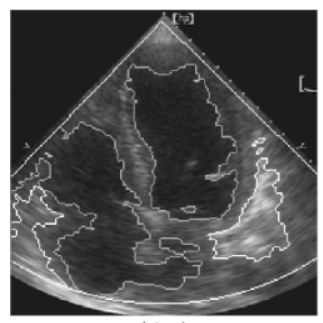

(A2)

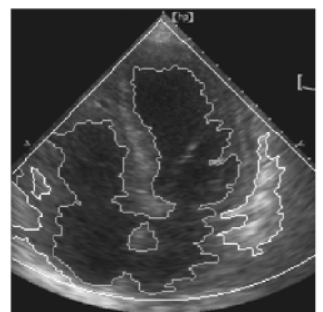

(A3)

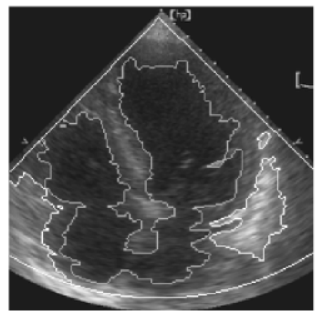

(A4)

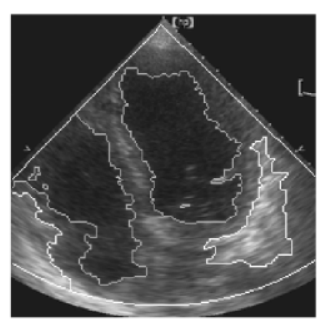

(B1)

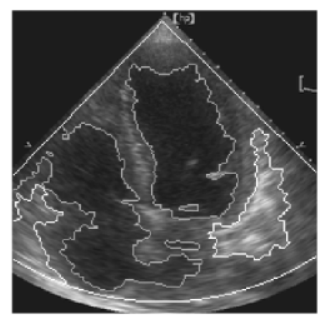

(B2)

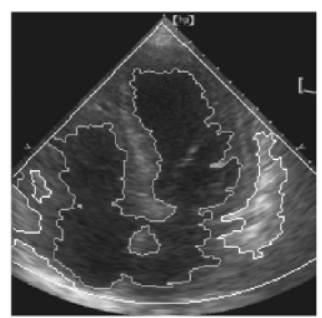

(B3)

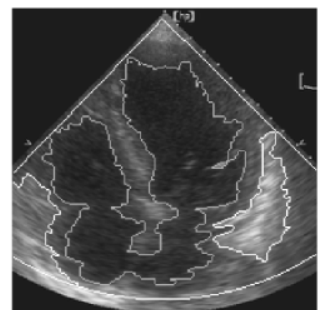

(B4)

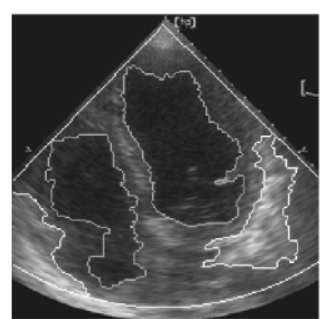

(C1)

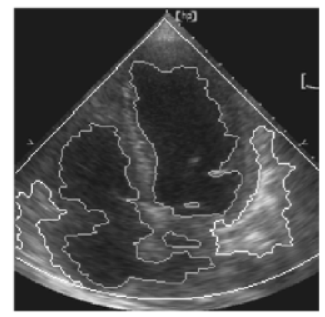

(C2)



(C3)

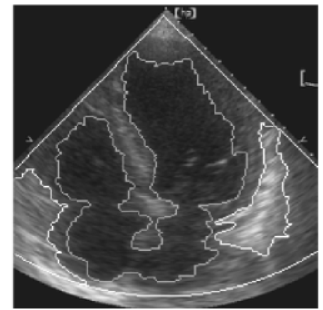

(C4)

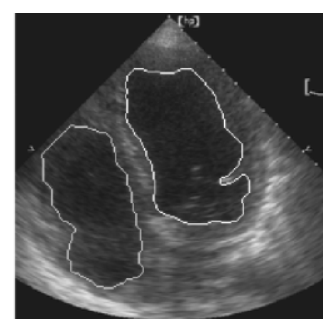

(D1)

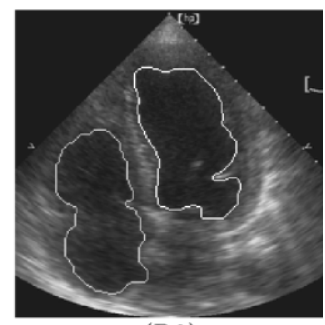

(D2)

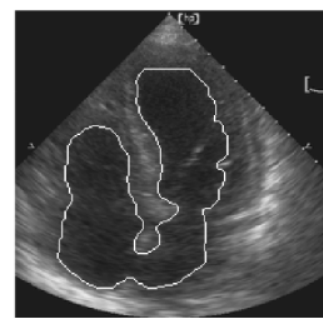

(D3)

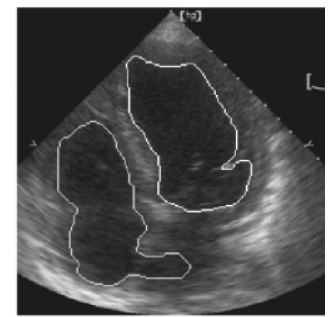

(D4)

Fig. 5. Segmentation results for three different values of the control parameter $a$ : $a=+\infty(\mathrm{A}), a=10$ (B) and $a=1$ (C). Segmentation results of Xiao et al. (2000) (D). Frames 1 and 2: systole, frames 3 and 4: diastole. 
segmentation process (Fig. 5C). As expected, the segmentation is better when both global and local statistics were included in the segmentation process. Our formulation of the problem allows us to control the effective contribution of each set of statistics by varying the parameter $a$. There is little difference between results $\mathrm{A}$ and $\mathrm{B}$, probably because in experiment B the global statistics are involved with a low weight $(10 \%$ of the weight of local statistics). This provides some insight into the importance of the parameter $a$.

An evaluation of the computer detection of the boundary of the left ventricle has been made. First we computed two classical measures (perimeter and area) on the 106 frames of the computergenerated and the hand-outlined boundaries. The results are summarized in Table 1. In particular, we can see that the perimeter results show us that little improvement is achieved when global statistics are involved and that Xiao's algorithm has the smallest perimeter error. However it is quite difficult to make a conclusion using only these two measures. Indeed, the perimeter error is highly related to the smoothness of the contours then to its localisation. Xiao's segmentation is smoother then the three results obtained with the proposed method. Moreover the area errors do not show any significant difference between the four algorithms.

Recently, a more applicable methodology for evaluation of boundary detection algorithm has been proposed (Chalana and Kim, 1997). This methodology uses essentially two distance measures: The Hausdorff distance (HD) and the average distance (AD). The HD between two curves is defined as the maximum of the distance to the closest point's (DCP) between the two curves. The DCP associates each point on both curves to a point on the other curve, and the HD finds the largest distance between the associated points. The AD is the average of all distances between the corresponding points between the curves. This effectively allows us to analyse the regional differences between the curves.

Table 2 shows the minimum, the maximum, the mean and the standard deviation of the two distances over all data sets (106 frames). Note that the HD does not show any significant difference between the performance of the three algorithms A, B and C. However the AD shows clearly that the third algorithm is better than the two others $(p<0.0001$ for the Friedman's rank test). Regarding the comparison between the proposed technique (results C) and Xiao's algorithm (results

Table 1

Perimeter and area error measures of the computer-generated segmentation of the left ventricle relative to measurement made by hand by a medical expert ${ }^{\mathrm{a}}$

\begin{tabular}{|c|c|c|c|c|}
\hline & $a=+\infty$ & $a=10$ & $a=1$ & Xiao et al. (2000) \\
\hline Perimeter, \% (pixel size) & $\begin{array}{l}\mu=-28.83 \\
\sigma=8.22\end{array}$ & $\begin{array}{l}\mu=-25.38 \\
\sigma=7.17\end{array}$ & $\begin{array}{l}\mu=-19.52 \\
\sigma=4.99\end{array}$ & $\begin{array}{l}\mu=-8.85 \\
\sigma=6.24\end{array}$ \\
\hline Area, $\%(\text { pixel size })^{2}$ & $\begin{aligned} \mu & =-6.29 \\
\sigma & =6.91\end{aligned}$ & $\begin{array}{l}\mu=-6.03 \\
\sigma=6.92\end{array}$ & $\begin{aligned} \mu & =-7.59 \\
\sigma & =6.71\end{aligned}$ & $\begin{array}{l}\mu=-6.41 \\
\sigma=8.06\end{array}$ \\
\hline
\end{tabular}

${ }^{a}$ The minus sign indicates that the estimation is larger than the medical expert's.

Table 2

Direct comparison of the computer-generated boundaries of the left ventricle to the hand-outlined by a medical expert (results in pixel)

\begin{tabular}{llllll}
\hline & & $a=+\infty$ & $a=10$ & $a=1$ & Xiao et al. (2000) \\
\hline \multirow{2}{*}{$\mathrm{HD}$} & $(\min , \max )$ & $8.06,23.53$ & $8.06,23.53$ & $8.48,22.56$ & $8.06,25.50$ \\
& $(\mu, \sigma)$ & $14.21,3.91$ & $13.63,3.74$ & $13.64,3.48$ & $16.35,4.49$ \\
$\mathrm{AD}$ & $(\min , \max )$ & $2.49,17.13$ & $2.45,15.80$ & $1.82,11.75$ & $2.64,12.40$ \\
& $(\mu, \sigma)$ & $8.78,3.09$ & $8.19,2.94$ & $5.96,2.20$ & $5.67,2.07$ \\
\hline
\end{tabular}




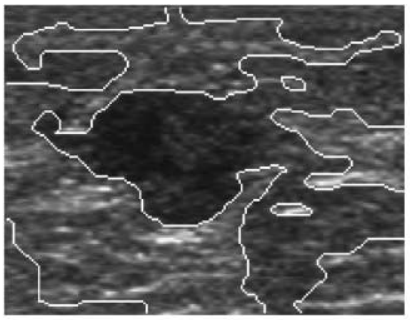

$n=2, \beta^{0}=0.3$

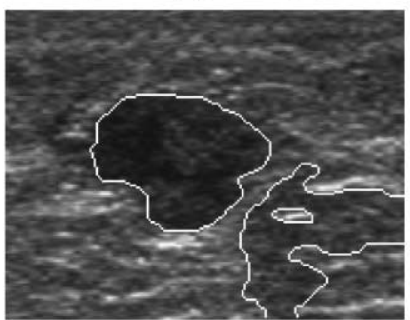

$n=3, \beta^{0}=0.3$

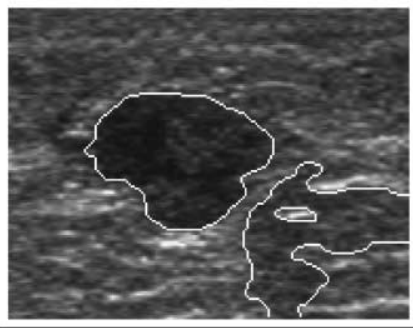

$n=4, \beta^{0}=0.3$

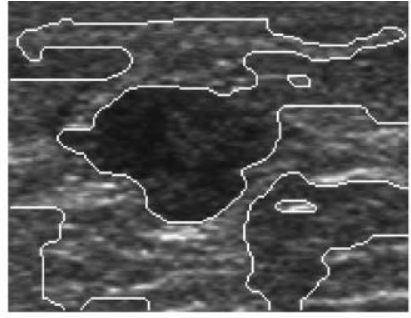

$n=2, \beta^{0}=0.4$

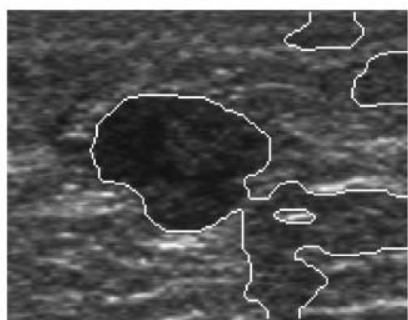

$n=3, \beta^{0}=0.4$

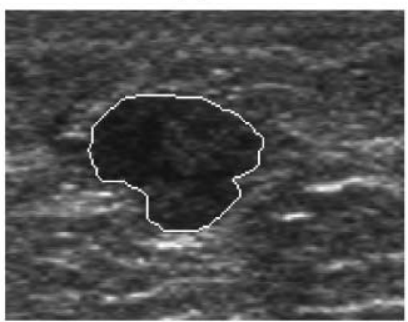

$n=4, \beta^{0}=0.4$

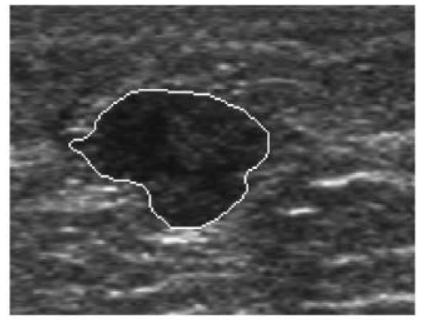

$n=2, \beta^{0}=0.4$

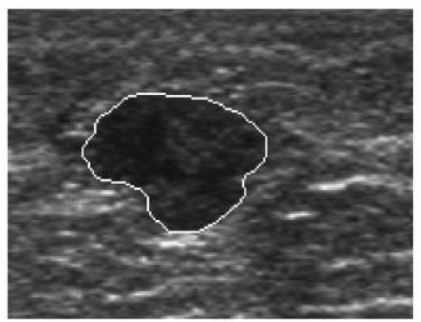

$n=3, \beta^{0}=0.4$

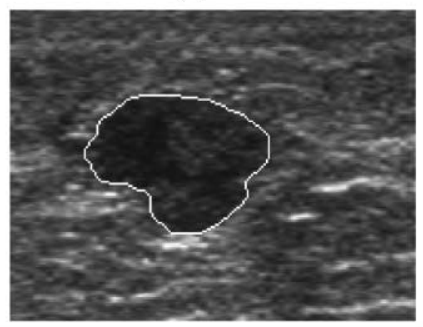

$n=4, \beta^{0}=0.4$

(a)

(b)

Fig. 6. Breast lesion segmentation with $k=2$ for different resolution level values $(n)$ of the DWT. All the results were obtained using three textural features calculated on the co-occurrence matrix (angular second moment, correlation and sum average). Results obtained with: $a=+\infty$ (i.e. energy function in (3)) (a); $a=b=1$ (b).

D), the HD distance shows a significant difference $(p<0.001)$, however the AD measure shows that the performance of the two algorithms are similar $(p>0.7)$. In other words, the average segmentation error of the left ventricle of the two techniques in comparison to the manual contours is relatively the same.

\subsection{Breast data}

Finally, we applied the new weighting function to the multiparametric model, and an example of segmenting breast ultrasound images is shown in Fig. 6. In this experiment we made use of the cooccurrence matrix method ${ }^{1}$ to calculate three textural parameters. In our previous work (Boukerroui et al., 1998), we have shown that a more robust lesion detection can be obtained when the textural features characterising pathological tissues are involved in the segmentation process. The

\footnotetext{
${ }^{1}$ The reader may find more details about the co-occurrence matrix method in (Haralick et al., 1973) and on the contribution of the textural features in (Boukerroui et al., 1998).
} 
three textural parameters used in this experiment were: angular second moment, correlation and sum average.

The aim of this experiment was to show that including the global statistics in the segmentation process, makes the segmentation result less parameter dependent. Fig. 6 shows the segmentation results of the fully adaptive algorithm for three different resolution level $(n)$ and for 2 values of the regularisation parameter $\beta^{0}$ (results (a)). We observe that the results of the fully adaptive algorithm depend on the number of the resolution levels of the DWT. The results are better for four levels than for a lower number of resolution levels. This result was expected as the approximation error of the image statistics by a Gaussian distribution decreases with the resolution levels in the pyramid (Ashton and Parker, 1995). However, the proposed approach converges almost to the same solution for the three values of the resolution level (see results (b)). This experiment offers a clear demonstration of the robustness of our algorithm.

\section{Conclusion}

In this paper, we have proposed an adaptive region segmentation algorithm based on global and local statistics in a Bayesian framework. The enhancement we developed can be regarded as a generalisation of our previous work (Boukerroui et al., 1998). The formulation of the segmentation problem allows us to control the effective contribution of the local and global statistics. The effect of the weighting introduced to control the adaptivity of the segmentation process has been illustrated on synthetic images, a long-axis echocardiographic sequences $(2 \mathrm{D}+\mathrm{T})$ and on $2 \mathrm{D}$ breast ultrasound images. Our experiments indicate that including the global statistics in an adaptive context improves significantly the segmentation results and the robustness of the algorithm.

The results obtained with our algorithm on echocardiographic data gives a good segmentation result when compared against boundaries of the left ventricle hand-outlined by a medical expert. However, a comparison of the segmentation re- sults with boundaries outlined by multiple observers would be an important future step to confirm this conclusion. It would be also interesting to associate our approach with high-level model-guided segmentation using "deformabletemplates" for instance, which include a priori knowledge about the expected object.

\section{Acknowledgements}

We gratefully acknowledge Guofung Xiao from Medical Vision Laboratory, Oxford University, for providing segmentation results on our data set for comparison.

\section{Appendix A}

In this appendix, we propose a different formulation of the weighting coefficients. The interpretation of this new expression of the weighting coefficient is easier. For each site $s$, we adopt the simplified following notation:

$$
\begin{aligned}
& W^{l}=\left(W_{s}^{x_{s}}\right)^{b} \in[0,1], \\
& \text { Dyn }=\max _{\text {all classes }}\left\{W^{l}\right\}-\min _{\text {all classes }}\left\{W^{l}\right\}, \\
& w^{l}=\frac{\varphi\left(W_{s}^{l}\right)}{N_{s}^{\varphi}} .
\end{aligned}
$$

Hence, from Eqs. (5)-(7), the different weighting coefficients of the different labels in the analysing window centred at the site $s$ are given by

$$
\begin{aligned}
w^{l} & =\frac{a \operatorname{Dyn}+W^{l}}{\sum_{l=1}^{\tilde{k}}\left(a \operatorname{Dyn}+W^{l}\right)} \tilde{k}, \\
& =1+\frac{W^{l}-\bar{W}}{a \operatorname{Dyn}+\bar{W}}, \quad \text { for } l=1, \ldots, \tilde{k}, \\
& =1+\Delta\left(W^{l}\right),
\end{aligned}
$$

where $\bar{W}$ is the mean value of $\left\{W^{l}, l=1, \ldots, \tilde{k}\right\}$.

The interpretation of the different weighting coefficients from equation (A.1) is much easier and we can note the following properties of the weighting coefficients: 


$$
\left\{\begin{array}{l}
w^{l}<1 \quad \text { if } W^{l}<\bar{W} ; \\
w^{l}>1 \quad \text { if } W^{l}>\bar{W} ; \\
\underbrace{\left|\Delta\left(W^{l}\right)\right|}_{a 1}<\underbrace{\left|\Delta\left(W^{l}\right)\right|}_{a 2} \text { if } a 1<a 2 ; \\
\lim _{a \rightarrow \infty} w^{l}=1 ; \\
\tilde{\tilde{k}} w_{l=1}^{l}=\tilde{k} .
\end{array}\right.
$$

\section{References}

Ashton, E.A., Parker, K.J., 1995. Multiple resolution Bayesian segmentation of ultrasound images. Ultrasonic Imaging 17 (2), 291-304.

Besag, B.J., 1974. Spatial interaction and the statistical analysis of lattice systems. J. Royal Statist. Soc. B 26 (2), 192-236.

Besag, B.J., 1986. On the statistical analysis of dirty pictures. J. Royal Statist. Soc. B 48 (3), 259-302.

Boukerroui, D., Basset, O., Baskurt, A., Gimenez, G., 2001. A multiparametric and multiresolution segmentation algorithm of 3D ultrasonic data. IEEE Trans. Ultrason. Ferroect. Freq. Control 48 (1), 64-77.

Boukerroui, D., Basset, O., Guérin, N., Baskurt, A., 1998. Multiresolution texture based adaptive clustering algorithm for breast lesion segmentation. Euro. J. Ultrasound 8 (2), 135-144.

Bouman, C., Liu, B., 1991. Multiple resolution segmentation of textured images. IEEE Trans. Pattern Anal. Machine Intell. 13 (2), 99-113.

Chalana, V., Kim, Y., 1997. A methodology for evaluation of boundary detection algorithm on medical images. IEEE Trans. Med. Imaging 16 (1), 642-652.

Geman, D., 1990. Random fields and inverse problems in imaging. In: Hennequin, P.L. (Ed.), Ecole d'été de probabilités de Saint-Flour XVIII, Lecture Notes in Mathematics, Vol. 1421. Springer-Verlag, Berlin, pp. 161-172.
Haralick, R.M., Shanmugam, K., Dinstein, I., 1973. Textural features for image classification. IEEE Trans. System Man Cybernet. 3 (6), 610-621.

Heitz, F., Perez, P., Bouthemy, P., 1994. Multiscale minimization of global energy functions in some visual recovery problems. CVGIP: Image Understanding 59 (1), 125-134.

Hughes, D.I., Duck, F.A., 1997. Automatic attenuation compensation for ultrasonic imaging. Ultrasound Med. Biol. 23, 651-664.

Kervrann, C., Heitz, F., 1994. Segmentation non-supervisée des images naturelles texturées: approche statistique. Trait. signal $11(1), 31-41$.

Kirkpatrick, S., Gelatt, C., Vecchi, M., 1983. Optimization by simulated annealing. Science 220, 671-680.

Mulet-Parada, M., Noble. J.A., 1998. 2D + T Boundary detection in echocardiography. In: Proc. Internat. Conf. on Med. Image Comp. and Computer Assisted Intervention MICCAI, Cambridge, MA, USA, pp. 186-196.

Mulet-Parada, M., Noble, J.A., 2000. 2D + T acoustic boundary detection in echocardiography. Med. Image Anal. 4, 2130 .

Muzzolini, R., Yang, Y.-H., Pierson, R., 1993. Multiresolution texture segmentation with application to diagnostic ultrasound images. IEEE Trans. Med. Imaging 12 (1), 108-123.

Pappas, T.N., 1992. An adaptive clustering algorithm for image segmentation. IEEE Trans. Signal Process. SP-40 (4), 901914.

Unser, M., 1995. Multigrid adaptive image processing, In: Proc. Int. IEEE Conf. on Image Process. ICIP, Vol. 1. Washington DC, USA, pp. 49-52.

Vemuri, B.C., Rahman, S., Li, J., 1995. Multiresolution adaptive K-means algorithm for segmentation of brain MRI. In: Intertnat. Comput. Sci. Conf. On Image Analysis and Computer Graphics, Hong Kong, pp. 347-354.

Wells, W.M., Grimson, E.L., Kikinis, R., Jolesz, F.A., 1996. Adaptive segmentation of MRI data. IEEE Trans. Med. Imaging 15 (4), 429-442.

Xiao, G., Brady, J.M., Noble, J.A., Zhang, Y., 2000. Contrast enhancement and segmentation of ultrasound images-a statistical method. In: Proc. SPIE Med. Imaging Image Processing, pp. 1116-1125. 